\title{
El desarrollo financiero en épocas de crisis económica - caso de estudio América Latina: Argentina, Chile y Colombia
}

\section{Financial development in times of economic crisis- case study Latin America: Argentina, Chile and Colombia}

\author{
Martha Isabel Amado - Piñeros ${ }^{1}$ \\ ${ }^{1}$ Grupo de investigación Quipus, Universidad o institución: Fundación Universitaria del Área Andina, Colombia, \\ ORCID: https://orcid.org/0000-0002-6029-3357. E-mail: mamado7@ areandona.edu.co
}

Cómo citar: Amado - Piñeros, M. I. (2020). El desarrollo financiero en épocas de crisis económica - caso de estudio América Latina: Argentina, Chile y Colombia. Revista Científica Profundidad Construyendo Futuro, 13(13), 21-30. https://doi.org/10.22463/24221783.2547

Recibido: 15 de Abril de 2020 / Aprobado: 15 de Julio de 2020

\begin{abstract}
Resumen
Las regiones que hacen parte de un sector geográfico deben postular escenarios de protagonismo autóctono entre sus líderes y gestores de cambio, no solo desde lo local sino desde lo financiero con el fin de lograr que se consoliden desarrollos financieros a largo plazo. Colombia, Chile y Argentina se perfilan como ejemplos de crisis a lo largo de varias décadas y se han visto condicionadas sus economías y desarrollos financieros a dichos momentos históricos. El presente artículo tenía como objetivo abordar las principales medidas e indicadores que han postulado los gobiernos de Colombia, Argentina y Chile frente al manejo de las finanzas. El proceso metodológico fue documental tomando los informes la CEPAL, Banco Mundial, OCDE y además estamentos internos a cada región comentada. Los informes de economía, estudios de crecimiento serán la fuente primaria de revisión, dentro de los resultados obtenidos esta la presentación de análisis sectoriales e ideas rescatadas de dichos organismos de cooperación entre Colombia, Argentina y Chile como pilares de la economía local y regional. Se busca servir de base para futuros profesionales de la Contaduría en el entorno histórico para que de manera integral se comprendan escenarios globalizados de la región latinoamericana.
\end{abstract}

Palabra clave: Banco Mundial, CEPAL, Crisis económica, Desarrollo financiero, ONU, Organización para la Cooperación y el Desarrollo Económicos.

\begin{abstract}
The regions that are part of a geographical sector must postulate scenarios of autochthonous protagonism among their leaders and change managers, not only from the local but also from the financial point of view, in order to consolidate long-term financial developments. Colombia, Chile and Argentina are emerging as examples of crises over several decades and their economies and financial developments have been conditioned to these historical moments. The objective of this article was to address the main measures and indicators that the governments of Colombia, Argentina and Chile have postulated regarding the management of finances. The methodological process was documentary, taking reports from ECLAC, the World Bank, OECD and also internal levels to each commented region. The economy reports, growth studies will be the primary source of review. Among the results obtained is the presentation of sectoral analyzes and ideas rescued from said cooperation organizations between Colombia, Argentina and Chile as pillars of the local and regional economy. It seeks to serve as a base for future accounting professionals in the historical environment so that globalized scenarios of the Latin American region are fully understood.
\end{abstract}


Keywords: World Bank, CEPAL, Economic crisis, financial Development, ONU, Organization for Economic Cooperation and Development.

\section{Introducción}

Los modelos de desarrollo financiero han dependido del crecimiento en los ingresos, en los reportes obtenidos por actividades de inversión, operación y financiación. Debido al confinamiento y el aumento del desempleo en varias regiones del mundo por la pandemia, muchas empresas no han incrementado porcentajes de ventas y por lo tanto las variables de desarrollo financiero se han visto disminuidas a valores negativos.

Muchos aspectos conocidos a nivel económico surgen de las dinámicas entre los individuos. Sin embargo, al contemplar dicho desarrollo desde una mirada financiera, se desprenden conexiones locales y regionales, que obligan a tejer redes, a delegar responsables y a mitigar riesgos.

Las crisis económicas guardan escenarios de repensar, de modificar ciertas dinámicas y comportamientos llevados hasta el momento y nunca se presentan avisando con antelación. Dependen y condicionan al mismo tiempo desarrollos financieros, políticos, culturales, por lo que las regiones en las cuales sucede albergan objetivos disimules entre los sujetos que las representan.

El presente escrito pretende analizar solo 3 sectores y tipos de economías latinoamericanas como lo son Argentina, Chile y Colombia frente a las crisis económicas vividas, la posibilidad de fortalecimiento de sus finanzas.

Las tres económicas son vistas con las cifras de Banco Mundial, la CEPAL, la OCDE y la ONU. De ellas se realizan análisis de la historia reciente $\mathrm{y}$ algunas ideas $\mathrm{y}$ preguntas que pueden dar pie para futuras preguntas de investigación. Con este artículo se pretende mostrar como al depender de unas variables netamente financieras, los desarrollos de las naciones tienen una visión parcializada, no integradora.

\section{Marco Teórico}

\subsection{Banco Mundial}

En el comunicado de prensa N. ${ }^{\circ}$ 2020/175/SPJ del Banco Mundial emitido el 22 de abril de 2020 aduce que la llegada de remesas a América Latina y el Caribe creció el $7,4 \%$ en 2019 y alcanzó los USD 96000 millones, aunque el aumento fue dispar en los distintos países de la región. En Brasil, Guatemala y Honduras, fue superior al $12 \%$ en 2019. En Colombia, Ecuador, Nicaragua y Panamá, las remesas crecieron más del $6 \%$, mientras que las enviadas a Bolivia y Paraguay se redujeron un 3,8 \% y $2,2 \%$ respectivamente. Se estima que, en 2020, la llegada de remesas a la región se reducirá un $19,3 \%$.

Por otro lado, presentan el costo de las remesas afirmando lo siguiente: el costo promedio de enviar USD 200 a la región fue de 5,97 \% en el primer trimestre de 2020. En el contexto de la crisis de la COVID-19, los costos de transferir remesas a la región podrían elevarse debido a las dificultades operativas que enfrentan los proveedores de estos servicios (cierre de agentes y de oficinas, acceso al dinero en efectivo, tipo de cambio, seguridad) y al cumplimiento de las normas contra el lavado de activos y el financiamiento del terrorismo. 
Para el Banco Mundial, la corrección financiera es clave en momentos de crisis, por tal razón afirma: "Desde 2010, más de 55 países se han comprometido a implementar la inclusión financiera, y más de 30 de ellos han puesto en marcha o están preparando una estrategia nacional al respecto" (Banco Mundial, 2018)

Específicamente Chile, Argentina y Colombia presentan cifras en esta institución que enseguida se pretenden analizar. En un estudio sobre las tendencias econométricas se dan aportes sobre los modelos de evaluación que condicionan los crecimientos económicos en algunos sectores.

Lo que cambia con el tiempo es el comportamiento correcto de inclinación y cola (en oposición al promedio) de la distribución del daño. Esto puede explicarse utilizando el concepto de función de daño. Las funciones de daños se usan ampliamente en la literatura del Modelo de Evaluación Integrada para vincular los factores estresantes relacionados con el clima (por ejemplo, la velocidad del viento para ciclones tropicales o mareas de tormenta) con los daños. La caracterización de tales funciones plantea desafíos conceptuales y econométricos (24-27) que son específicos de los sectores económicos, escalas espaciotemporales y retroalimentaciones (p. Ej., Adaptación) bajo consideración. Sin embargo, las funciones obtenidas a partir de datos globales, regionales y locales son a menudo convexas y curvadas hacia arriba $(26,28 \Downarrow-30)$, probablemente debido a la exposición o la fragilidad de aumento no lineal (24). Basado en tal forma, un cambio simple en la distribución del estresante subyacente se traduce en un sesgo hacia la derecha y un engorde de la cola de la distribución del daño (daños mayores).

Y dentro de este escenario, Colombia en las perspectivas analizadas por el Banco Mundial aparece frente a la tasa del PIB con valores en crecimiento. (Banco Mundial, 2019, pág. 1)

En esta imagen se presenta un evidente crecimiento del PIB entre los años 2017 a 2021 y se estabiliza para el siguiente año. Sin embargo, esta situación cambiaria dados los indicadores posteriores a la repercusión del COVID 19.

\subsection{CEPAL}

Por otro lado, en el informe de la CEPAL, desde 1974, ha desarrollado un avance teórico alrededor de lo que entiende por desarrollo regional. Por desarrollo regional se entiende que hay un aumento en el bienestar de la región expresado por indicadores tales como ingreso per cápita, disponibilidad de servicios sociales y la adecuacidad de sus sistemas legales y administrativos. (p. 3).

Más adelante presenta 6 avances que a la época se dieron en términos globales:

Los más importantes entre éstos, son los siguientes:

1. El crecimiento regional resulta de un conjunto de decisiones tomadas dentro y fuera de la región, que llevan al comercio interregional.

2. El crecimiento regional como todo crecimiento económico, es acelerado por la especialización y la creación de economías de escala; éstos, a su vez, parcialmente determinan la estructura espacial resultante de los centros.

3. Los requerimientos necesarios para el crecimiento regional autosostenible son: una distribución adecuada del ingreso y la capacidad de minimizar los efectos de polarización ejercidos por la región primaria.

4. Dentro de la estructura de los centros, se puede observar una cierta jerarquía que se 
explica, por una parte, por las fuerzas administrativas, pero, por la otra (y principalmente), por el deseo de parte de productores y consumidores de minimizar los costos de transporte.

5. "El carácter del desarrollo regional es tal, que éste es un proceso de integración basado en las fuerzas económicas, sociales y administrativas. Infortunadamente, sin embargo, el rol de las fuerzas sociales no está bastante claro." (p. 16)

Termina su análisis argumentando que "los esfuerzos de la región dominante para mantener en la región más pobre una estructura socio-política que perpetúe el carácter subordinado de la última resultará eventualmente en vano.” (p. 19)

Pero dado que la historia continua y que el reporte luego de varios cambios de gobierno ha sufrido modificaciones, es importante analizar situaciones más actuales que las presentadas en ese inicio.

\subsection{OCDE}

En algunos comunicados de prensa que han surgido con efecto de las situaciones actuales, año 2020, la OCDE ha afirmado:

"Nos esforzaremos por ayudar a los Países Menos Adelantados y otros países con necesidades específicas mediante una respuesta coherente y coordinada en los ámbitos humanitario, del desarrollo y la paz". (OCDE, 2020, p. 2).

Esto significa que la intención está dada y los Gobiernos se han convocado en un solo objetivo. Siendo en entorno económico liderado por Chile y Argentina, el centro de este escrito, se presentan las cifras de análisis a continuación.
Para Argentina, las cifras son esperanzadoras para este año 2020, dado que presento diferentes niveles de crecimiento del PIB, tal como lo muestra la imagen adjunta.

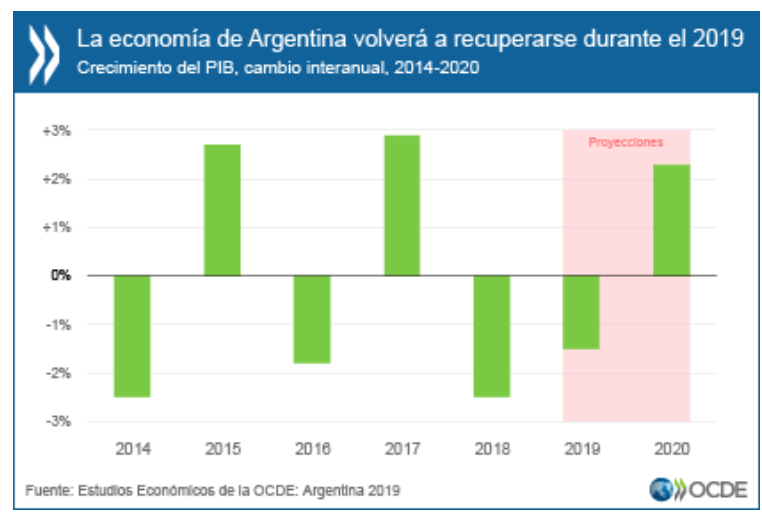

Figura 1. La economía de Argentina volverá a recuperarse durante el 2019.Fuente:(OCDE,2019,p. 1)

Para Chile, de acuerdo con el Estudio Económico de la OCDE 2018, para sostener el crecimiento, Chile debe diversificar su economía hacia actividades no relacionadas con los recursos naturales (OCDE, 2017, p. 23).

La figura que se presenta da cuenta de algunas ideas que se han dado en ese país para facilitar el comercio, como eje de la producción económica y del manejo de las finanzas en Chile. Algunas áreas de análisis son la gobernanza e imparcialidad, los procedimientos de apelación, entre otros. Manejados conjuntamente dichos indicadores, dan una visión global y comparativamente a toda Latinoamérica frente a un sector en especial. 


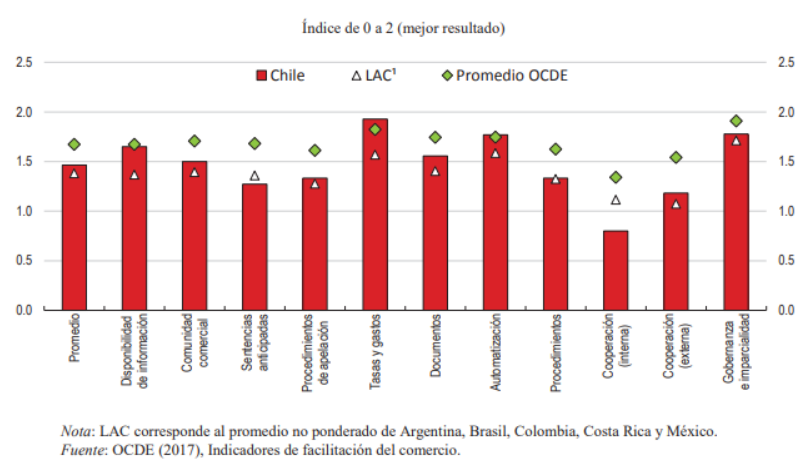

Figura 2. Margen para aumentar la facilitación del comercio en algunas áreas, 2017. Fuente: (OCDE, 2017 , p. 55)

De acuerdo con la gráfica, Chile se encuentra en iguales términos en tasas y gastos, así como en automatización frente al promedio de la OCDE, que contempla a países como Brasil, Colombia, Argentina, México y Costa Rica.

Para Colombia, según la OCDE se han logrado avances importantes en el frente social en las últimas décadas, gracias a la mejora del acceso a la educación y a las transferencias sociales. La esperanza de vida al nacer pasó de 60 años en 1970, a 70 años en 2016. Además, la pobreza disminuyó en 15 puntos porcentuales en el último decenio: del $42 \%$ en 2008 al $27 \%$ en 2018, según datos oficiales. (OCDE, 2019, p. 5) Y termina diciendo que es importante seguir llevando a cabo reformas estructurales para que Colombia converja hacia los niveles de vida de la OCDE y haga que su economía sea más incluyente.

Los índices Gini a nivel regional para el año 2015 plantean y miden hasta qué punto la distribución del ingreso (o, en algunos casos, el gasto de consumo) entre individuos $u$ hogares dentro de una economía se aleja de una distribución perfectamente equitativa. (OCDE, 2015, p. 10)
Esto muestra que en Colombia el departamento con mayor desigualdad es Chocó y el de menor desigualdad es Cundinamarca.

Así mismo, los países con menor índice Gini, es decir, menor niveles de desigualdad están ubicados en Europa como los países bajos y Asia como Japón. Además, presenta dentro de los análisis de investigación y desarrollo comparación entre países tal como se presenta en la siguiente imagen:

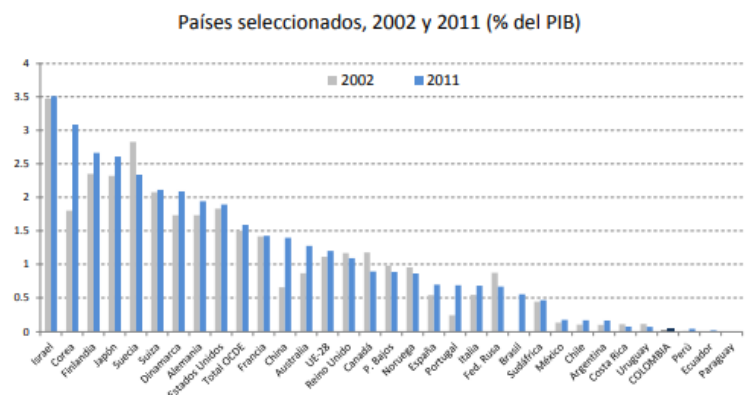

Figura 3. El gasto en I+D es mínimo en comparación con los países de la OCDE. Fuente (OCDE, 2015, p. 34)

Esto significa que Colombia en comparación con los países de la OCDE invierte menos en I+D quedando superada por Argentina y Chile y que países del sector asiático como Coreo e Israel son los líderes en dicha inversión.

\subsection{Naciones Unidas (ONU)}

Por parte de las Naciones Unidas como estamento gestor de políticas económicas con funciones de mantener la paz y seguridad internacionales, fomentar relaciones de amistad entre las naciones, lograr la cooperación internacional para solucionar problemas globales y servir de centro que armonice las acciones de las naciones, en países en desarrollo, el impulso financiero en épocas de crisis se dará con una economía 
dinámica además de ello debe ser inclusiva: "Para poder alcanzar los ambiciosos objetivos de la Agenda 2030 para el Desarrollo Sostenible, la economía mundial debe ser dinámica e inclusiva" (Naciones Unidas, 2019, p. 4)
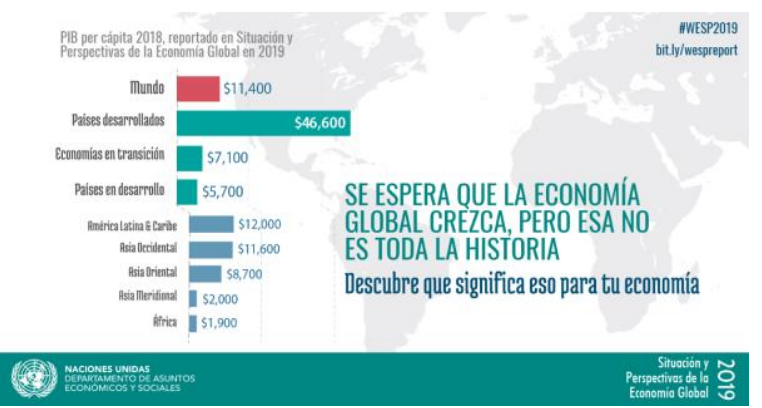

Figura 4. Fuente: (Naciones Unidas, 2019, p. 4)

De acuerdo con la imagen presentada, el Departamento de Asuntos Económicos y sociales de las Naciones Unidas mostró un crecimiento no tan alentador para los países de América Latina, dado que, comparados con países desarrollados, el crecimiento seria para el año 2019 casi de una cuarta parte.

Para Chile, y demás países, el Secretario General de la ONU también pidió que se diera una respuesta multilateral que representara, por lo menos, el $10 \%$ del producto interno bruto (PIB) mundial, a fin de dar la respuesta más eficaz a la crisis sin precedentes en el mundo. De esta manera, del desarrollo local se verá condicionado a delegar un porcentaje mayor del PIB en la reactivación financiera.

En el contexto argentino, según informes emitidos en periódicos globales, "la dinámica de la actividad económica en América Latina y el Caribe se da en un contexto en que la economía global ha disminuido su contribución al crecimiento de la región en 2019, situación que muy probablemente se mantendrá para el próximo año". (ONU, 2019, p. 1).

\section{Metodología}

\subsection{Tipo de Estudio}

El análisis documental presentado tomo como fuentes primarias informes de crecimiento y de economía de la OCDE, ONU, BM, y CEPAL para los años 2010 a 2020.

El tipo de estudio es descriptivo tomando como variables cuantitativas los índices de crecimiento, niveles de pobreza, gastos en investigación y desarrollo, porcentajes del PIB.

La información para esta reflexión contempla lo siguiente:

- El diseño de investigación.

- La muestra: informes económicos años 2010 a 2020

- Las restricciones o limitaciones: ninguna

- Las técnicas: análisis documental, fichas de trabajo y fichas bibliográficas

- Los procedimientos: descriptiva

- Los materiales: informes económicos, estadísticas sociales de las entidades.

- Las variables: pobreza, empleo, desigualdad, crecimiento económico, desarrollo financiero.

- El tratamiento estadístico: no probabilístico.

\section{Resultados}

Las economías que lideran regiones como la de Latinoamérica han presentado en forma conjunta resultados disimiles por intereses diversos. En las siguientes líneas se muestran resultados frente a la búsqueda de las soluciones a mercados más abiertos y solidos 
frente a tareas financieras que muestren datos confiables a las comunidades científicas.

Pedro Vuskovic en su artículo recibido el 29 de octubre de 2019 y aceptado el 12 de noviembre de 2019. Publicado originalmente en Cuadernos de la Realidad Nacional, núm. 5, septiembre de 1970, muestra que la "producción agropecuaria dejó de acompañar siquiera a la expansión del mercado interno, lo que, sumado a la pérdida o la lenta expansión del mercado externo, la llevó a registrar un ritmo de crecimiento extremadamente bajo". (p. 254).

Los eventos financieros no están marcados por jurados únicos, por evaluadores invisibles; los protagonistas acá son los usuarios de la información, pero que distan mucho de ser doctos en la materia, disciplinados en la lectura, como es el caso de pequeños y medianos empresarios.

Hacia ellos precisamente están focalizados los esfuerzos de préstamos, ayudas humanitarias. En ese sentido, entidades con mayor talante social como la ONU, que en contradicción con la CEPAL, BM y la OCDE los cuales persiguen fines netamente lucrativos, han permeado escenarios políticos, culturales en los cuales comunidades disimiles a nivel regional como lo son Argentina, Chile y Colombia, aumentando las diferencias entre los que tiene $\mathrm{y}$ los que no poder adquisitivo.

Los informes económicos que han emitidos estas entidades buscan explicar comportamientos a nivel local, regional e internacionales, lo cual implica tener variables de control frente a comunidades, planes de Estado y consecuencias de todo tipo, entre ellas las financieras.
Los efectos de desarrollo a nivel regional, tomando como centro de análisis a los tres países objeto de estudio en este artículo, están inversamente relacionados con la aparición de crisis de salud y económica, pero no son la razón fundamental para las pretensiones que se quieren y las visiones de mejoras que organismos de control y vigilancia han dispuesto como metas a cumplir.

La siguiente tabla muestra los índices de pobreza para los países latinos que se tenían hasta 2014.

Tabla 1. Índices de pobreza países latinos

\begin{tabular}{lc}
\hline \multicolumn{1}{c}{ País } & Índice \\
\hline Argentina & $33,20 \%$ \\
\hline Colombia & $28,90 \%$ \\
\hline México & $27,50 \%$ \\
\hline Bolivia & $25,90 \%$ \\
\hline Ecuador & $23,60 \%$ \\
\hline Perú & $20,10 \%$ \\
\hline Paraguay & $18,80 \%$ \\
Brasil & $18,10 \%$ \\
\hline Chile & $11,70 \%$ \\
\hline Uruguay & $6,70 \%$ \\
\hline
\end{tabular}

Nota. Índice de pobreza en Latino América Fuente: Datos suministrados por el Banco mundial en 2014.

Según la fuente, Argentina era el más pobre con un $33 \%$ seguido de Colombia.

Luego de la revisión documental se puede establecer correlación inversa entre el crecimiento económico y la disminución de los índices de pobreza. Para el BM, las proyecciones de crecimiento del PIB para los años 2020 y 2021 están por el orden de $-2 \%$ a $3,4 \%$ para Colombia; Chile de $-3 \%$ a $4,8 \%$ y Argentina de $-5,2 \%$ a $2,2 \%$.

En términos relativos, Colombia aumenta 5,4\%, Chile 7,8\% y Argentina 7,4\%. Así mismo, las crisis generadas en estos tres 
países han traído consigo consecuencias en sectores de las empresas de manera integral.

La crisis mexicana se propagó a países como Venezuela, Brasil, Chile y Argentina, lo que se conoció como el efecto tequila. En 1999, Colombia pasó por una profunda crisis financiera originada por el otorgamiento excesivo de préstamos de vivienda. Hoy las crisis muestran que el crecimiento económico en América Latina, genera un mayor estímulo para solicitar créditos, que para ahorrar.

En tiempos de pandemia como el actual generado desde 2019, según reportes de entidades gubernamentales, estaría más marcado el efecto de menos ingresos, mayor aceleración de mercados tecnológicos frente a la aparición de valores de desempleo cada vez mayores.

Los distintos estudios del personal afiliado a sectores específicos de la economía han mostrado así mismo que los consumos en tarjetas de crédito y compras online han aumentado. De esta manera el crecimiento económico en América Latina, genera un mayor estímulo para solicitar créditos, que para ahorrar.

El Gobierno colombiano tiene estimado tomar deuda con los mercados internacionales por US\$5.540 millones durante el segundo semestre del 2020 para atender las necesidades de gasto del país. En ese sentido, al aumentar la deuda, se esperaría que factores como el desempleo logren disminuir.

El ritmo y el tipo de crecimiento económico en los países de bajos ingresos pone en peligro los esfuerzos para reducir la pobreza y mejorar las condiciones de trabajo. En ese sentido, las 3 economías trabajadas en el presente artículo han demostrado tener bajos ingresos por cuenta propia, debiendo solicitar ayudar externas, que se constituyen en nuevas deudas a larguísimo largo plazo.

Todo lo anterior refleja la imposibilidad actual de auto-sostenimiento y de una continua crisis en círculo vicioso frente a sus pretensiones de cumplimiento de un desarrollo financiero sostenible.

\section{Conclusiones}

Poner de acuerdo variables financieras es complejo en economías y culturas con diferencias notorias.

Las finanzas son el motor de procesos económicos que reflejan el manejo interno del dinero dentro de una población.

Las poblaciones que están insertas en un sistema económico y logran encauzar dentro de sus políticas de estado vínculos comerciales que contemplen escuchar, participar y proponer con vecinos inmediatos o indirectos contemplan un sistema financiero más sólido que aquellas que manejen sus políticas de manera independiente.

Colombia, Chile y Argentina tienen mucho que aprender de escenarios europeos y asiáticos que han logrado estandarizar modelos de desarrollo para equilibrar índices como el Gini y un manejo de su investigación y desarrollo.

El COVID que se generó desde finales de 2019 ha sido una oportunidad de regenerar condiciones de manejo económico y de lazos empresariales con condiciones nuevas para muchas economías y de las cuales los países en desarrollo en mayoría no estaban preparados. 
Las finanzas impulsan el desarrollo de las naciones siempre y cuando haya medidas macroeconómicas que impulsen todos los sectores, entre ellos y como principal motor está el sector de investigación y desarrollo, pero así mismo el educativo y de salud deben trascender a mejores procesos, más organizados y propositivos.

Las cadenas de valor empresariales a nivel de finanzas dependen de los controles internos y externos que se hagan a los recursos financieros, descritos en las políticas contables y fiscales que se establezcan en manuales, notas a los estados financieros, informes de gestión, entre otras.

Las entidades como la ONU, BM, CEPAL y OCDE con procesos diferenciados y objetivos muy particulares ven de manera común que las regiones pueden fortalecer sus áreas de finanzas siempre y cuando trabajen conjuntamente en beneficio de sus comunidades y los lideres planteen planes de gobierno a más largo plazo, no por temporadas de 4 o 6 años.

\section{Referencias}

Banco Mundial. (20 de Abril de 2018). La inclusión financiera es un factor clave para reducir la pobreza e impulsar la prosperidad. Obtenido de https://www.bancomundial.org/es/top ic/financialinclusion/overview

Banco Mundial. (2019). Colombia en cifras. Obtenido de https://datos.bancomundial.org/pais/c olombia

Banco Mundial (2020). The economy in the time of covid-19. Semiannual report of the latin america and caribbean region, Abril de 2020.

CEPAL. (1974). Teoría del desarrollo regional: un intento de síntesis. Obtenido de https://repositorio.cepal.org/bitstream /handle/11362/33638/S7400534_es.p df? sequence $=1$

Naciones Unidas. (21 de enero de 2019). Situación y perspectivas de la economía mundial en 2019: Resumen ejecutivo. Obtenido de https://www.un.org/development/des a/dpad/publication/situacion-yperspectivas-de-la-economiamundial-en-2019-resumen-ejecutivo/

OCDE. (Enero de 2015). Colombia políticas prioritarias para un desarrollo inclusivo. Obtenido de https://www.oecd.org/about/publishi ng/colombia-politicas-prioritariaspara-un-desarrollo-inclusivo.pdf

OCDE. (6 de Diciembre de 2017). Estudio Económico del OCDE de Chile 2018. Obtenido de https://www.oecd.org/economy/surve ys/Chile-2018-OECD-economicsruvey-Spanish.pdf

OCDE. (27 de Marzo de 2019). La OCDE afirma que Argentina necesita más reformas para conseguir una economía más fuerte e inclusiva. Obtenido de http://www.oecd.org/newsroom/laocde-afirma-que-argentina-necesitamas-reformas-para-conseguir-unaeconomia-mas-fuerte-e-inclusiva.htm 
OCDE. (24 de Octubre de 2019). Presentación del Estudio Económico de la OCDE sobre Colombia 2019. Obtenido de https://www.oecd.org/about/secretary -general/economic-survey-ofcolombia-october-2019-sp.htm

OCDE. (9 de Abril de 2020). La pandemia mundial del COVID-19. Obtenido de http://www.oecd.org/dac/developmen t-assistance-committee/DeclaracionCAD-La-Pandemia-mundial-delCOVID-19.pdf

ONU. (11 de Noviembre de 2019). La economía de América Latina continúa estancada y las perspectivas apuntan a un bajo crecimiento. Noticias ONU, pág. 2. 